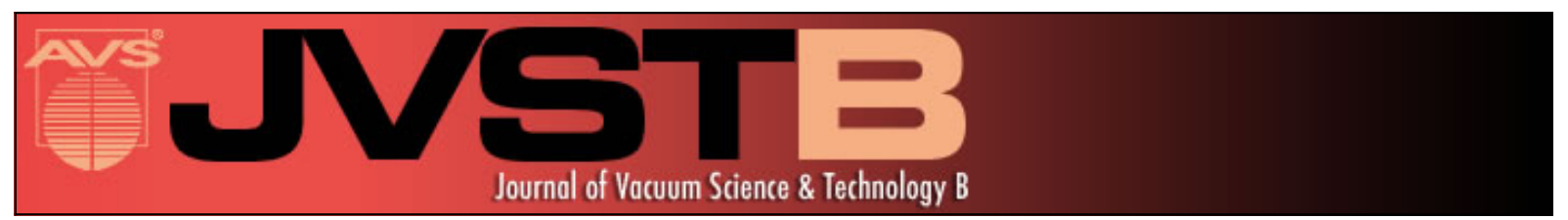

\title{
Density-tunable non-close-packed monolayer of silica nanospheres prepared by single-step freeze-drying
}

Cong Feng and Hoi Wai Choi

Citation: Journal of Vacuum Science \& Technology B 32, 051805 (2014); doi: 10.1116/1.4895037

View online: http://dx.doi.org/10.1116/1.4895037

View Table of Contents: http://scitation.aip.org/content/avs/journal/jvstb/32/5?ver=pdfcov

Published by the AVS: Science \& Technology of Materials, Interfaces, and Processing

\section{Articles you may be interested in}

High capacity cobalt boride prepared via vacuum freeze-drying method and used as anode material for alkaline secondary battery

J. Renewable Sustainable Energy 5, 021401 (2013); 10.1063/1.4798422

Surfactant doped silica aerogels dried at supercritical pressure

AIP Conf. Proc. 1512, 210 (2013); 10.1063/1.4790985

Room-temperature ferromagnetism in Co-doped $\mathrm{CeO} 2$ nanospheres prepared by the polyvinylpyrrolidoneassisted hydrothermal method

J. Appl. Phys. 112, 113904 (2012); 10.1063/1.4766273

Sol-gel NiFe2O4 nanoparticles: Effect of the silica coating

J. Appl. Phys. 111, 103911 (2012); 10.1063/1.4720079

Experimental Study of influence on The Moving of Sublimation Interface by Precooling Rate and Drying Temperature During Freeze-drying

AIP Conf. Proc. 914, 113 (2007); 10.1063/1.2747419

\section{HIDEN}

\section{Instruments for Advanced Science} w www.HidenAnalytical.com E info@hiden.co.uk CLICK TO VIEW our product catalogue

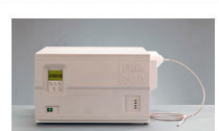

Gas Analysis

dynamic measurement of reaction gas steans catalysis and thermal analysis molecular beam studies

, dissolved species probes

fermentation enuronmental and ecological studies

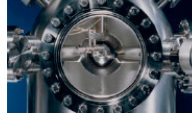

Surface Science

UHVTPD

SIMS emental imaging - surface mapping

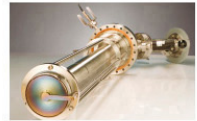

Plasma Diagnostics plasma source characterization etch and deposition process reaction , analysis of neutral and redical species

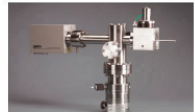

Vacuum Analysis , partial pressure measurement and contro of process gases reactive sputter process contro vacuum diagnostics Teum coatin prosesmonitorin 


\title{
Density-tunable non-close-packed monolayer of silica nanospheres prepared by single-step freeze-drying
}

\author{
Cong Feng and Hoi Wai Choi ${ }^{\mathrm{a})}$ \\ Department of Electrical and Electronic Engineering, The University of Hong Kong, Pokfulam Road, \\ Hong Kong
}

(Received 7 June 2014; accepted 26 August 2014; published 10 September 2014)

\begin{abstract}
The formation of density-tunable non-close-packed (ncp) monolayers of silica nanospheres via freeze drying is demonstrated. Monolayers of silica spheres with diameters of 120, 490, and $990 \mathrm{~nm}$ are achieved through a single-step freeze dry process. The densities of the spheres can be tuned by adjusting either the volume fraction of the spheres or the settling time before freezing. Issues involving defect-formation and defect-reduction are investigated. The experimental data suggest that this freeze drying method represents an efficient and robust way for achieving ncp monolayers of nanoparticles. (C) 2014 American Vacuum Society.
\end{abstract}

[http://dx.doi.org/10.1116/1.4895037]

\section{INTRODUCTION}

Nanosphere lithography (NSL) is a nanopatterning technique that uses self-assembled monolayers of nanospheres as a lithographic masking layer, which has been widely studied and applied in recent years owing to its simplicity and costeffectiveness. ${ }^{1-3}$ The key process of NSL is the formation of the mask from spherical beads (polystyrene and silica being the most common materials) that are initially in colloidal form; this mainly relies on the self-assembly phenomenon during evaporation of the dispersant. ${ }^{4,5}$ Till now, several large-area coating methods have been proposed, such as dip-coating, ${ }^{6}$ spin-coating, ${ }^{7}$ and the Langmuir-Blodgett method, ${ }^{8}$ producing hexagonal-close-packed (hcp) nanopatterns after self-assembly. However, for applications such as sensing or surface plasmon resonance, a non-close-packed (ncp) monolayer is often preferred. ${ }^{9,10}$ To achieve an ncp monolayer, post-treatment of an hcp monolayer is the most commonly adopted approach, including pattern reversal and sphere shrinkage. ${ }^{11-13}$ A main disadvantage of these methods is that the particle density is predefined and limited by the original hcp monolayer. The difficulty of forming an ncp monolayer directly arises from the dispersant, which is deionized (DI) water under most circumstances. During the drying process, the evaporation of water pulls the spheres into close-packed arrangements. By dispersing silica spheres in ethoxylated trimethylolpropane triacrylate monomer (ETPTA), Jiang et al. proposed a modified spin-coating process to achieve an ncp pattern. ${ }^{14,15}$ However, plasma posttreatment is required for subsequent removal of the ETPTA. Lohmüller et al. solved this problem from a different perspective by using sublimation drying (also known as freeze drying) technology instead of conventional evaporative drying. ${ }^{16}$ They reported ncp monolayer formation by surface charge-directed deposition, followed by a freeze drying process to prevent aggregation of the spheres during drying. In this work, the freeze drying approach for ncp coating is further explored; it was found that the coating step can be

${ }^{a)}$ Electronic mail: hwchoi@hku.hk naturally integrated into the drying process. A single-step ncp monolayer coating method is thus developed. Densitytunable ncp monolayers of silica spheres with diameter ranging from 120 to $990 \mathrm{~nm}$ have been obtained.

\section{EXPERIMENT}

Silica $\left(\mathrm{SiO}_{2}\right)$ spheres without surface modification suspended in DI water are used in this work. Spheres with diameters of $990 \pm 20 \mathrm{~nm}$ and $490 \pm 20 \mathrm{~nm}$ are acquired from Thermo Scientific, while the $120 \pm 40 \mathrm{~nm}$ spheres are acquired from Bangs Laboratories. The substrates are metal-organic chemical vapor deposition grown III-nitride light emitting diode wafers on 2-in. c-plane sapphire substrates. The wafers are ultrasonically cleaned in acetone, ethanol, and deionized water for $5 \mathrm{~min}$ sequentially prior to coating. The freeze-dry setup consists of a liquid nitrogencooled cryostat with temperature control in the range of $77-550 \mathrm{~K}$.

Schematic diagrams illustrating the freeze drying process are shown in Figs. 1(a)-1(d). Samples $(5 \times 5 \mathrm{~mm})$ diced from the wafers are placed horizontally onto the cold finger of the cryostat in a vacuum chamber. Ten microliters of the sphere suspension is dripped onto the wafer using a micropipette and smeared to cover the entire surface, giving a $\sim 0.4 \mathrm{~mm}$-thick water layer [Fig. 1(a)]. The wafer is kept still at room temperature and atmospheric ambient for several minutes to let the spheres partly settle down to the wafer [Fig. 1(b)]. The sample is then cooled down to $250 \mathrm{~K}$ and maintained for $5 \mathrm{~min}$, allowing the water to totally freeze [Fig. 1(c)]. Up to this stage, the chamber remains at atmospheric pressure. Subsequently, the chamber is pumped down to 1 mbar to facilitate sublimation, until all the ice has sublimated. With the pumping maintained, the temperature is gradually raised to just above room temperature and held for $5 \mathrm{~min}$; this is to avoid condensation of water vapor onto the substrate during venting. Finally, the chamber is vented, and an ncp monolayer can be observed to be formed on the surface of the wafer [Fig. 1(d)]. 
a)

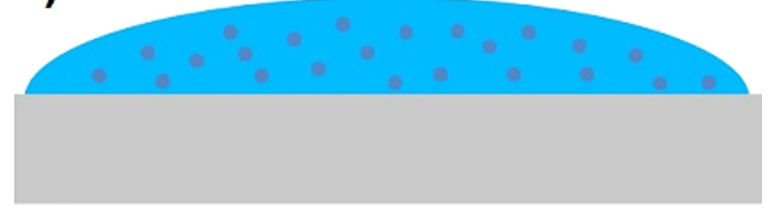

b)

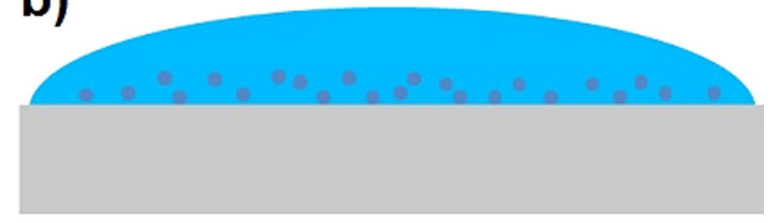

c)

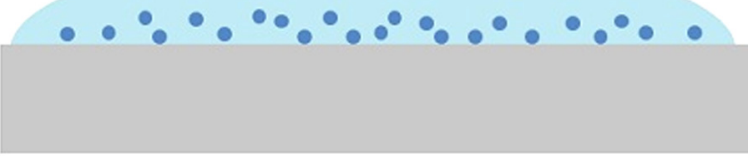

d)

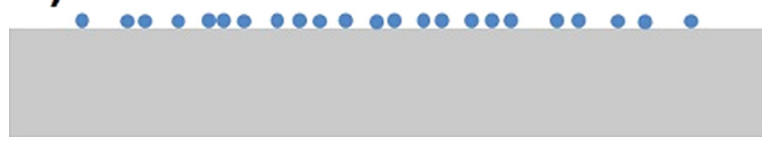

FIG. 1. (Color online) Schematic diagrams depicting the process of the freeze-dry coating. (a) A drop of sphere suspension is dripped onto the wafer. (b) After settling, the spheres sediment onto the wafer. (c) The water layer freezes into ice, fixing the locations of spheres. (d) The ice is freezedried, leaving ncp nanospheres on the wafer.

\section{RESULTS AND DISCUSSION}

\section{A. Coating mechanism}

There are two processes resulting in the formation of the final coating, the first one of which is the deposition of spheres before freezing, mainly driven by gravity. The other takes place during the freeze drying process. Ideally, as the ice sublimates, the remaining spheres are gradually pulled to the wafer surface. However, experiment results indicate that due to uneven ice sublimation, spheres further away from the wafer move not only vertically but also laterally, leading to the formation of aggregations. This phenomenon is closely linked to the formation of defects and will be discussed separately in a later. At this stage, the first deposition process is analyzed.

Consider spheres without surface charges, whose motion in water is driven mainly by four forces: buoyancy $\left(\mathrm{F}_{\mathrm{B}}\right)$, gravity $\left(\mathrm{F}_{\mathrm{g}}\right)$, drag $\left(\mathrm{F}_{\mathrm{d}}\right)$, and Brownian $\left(\mathrm{F}_{\mathrm{B}}\right)$. As the drag force is always a resistance that slows down the motion, the deposition depends on the competition between the three other forces. Note the densities of the silica spheres are around $2.2 \mathrm{~g} / \mathrm{cm}^{3}$. If the Brownian force is relatively small compared to the other two forces, deposition will be evident. On the other hand, if it is comparable to the other two forces, Brownian motion will dominate. To evaluate the deposition rate as a function of the sphere dimension, the motion of 10000 silica spheres in a $0.4 \mathrm{~mm}$-thick water layer is simulated. The respective forces applied upon a sphere are described by the following equations:

$$
\mathrm{F}_{\mathrm{B}}=\mathrm{g} \rho_{\mathrm{w}} \mathrm{V},
$$

where $\mathrm{g}$ is the gravitational acceleration, $\rho_{\mathrm{w}}$ the density of water, and $V=4 / 3 \pi r^{3}$ the volume of the sphere;

$$
\mathrm{F}_{\mathrm{g}}=\mathrm{g} \rho_{\mathrm{s}} \mathrm{V},
$$

where $\rho_{\mathrm{s}}$ is the density of silica;

$$
\mathrm{F}_{\mathrm{d}}=1 / 2 \rho_{\mathrm{w}} \mathrm{v}^{2} \mathrm{C}_{\mathrm{D}} \mathrm{A},
$$

where $\mathrm{v}$ is the velocity, $\mathrm{C}_{\mathrm{D}}$ the drag coefficient, and $\mathrm{A}=\pi \mathrm{r}^{2}$ the cross-sectional area.

The Brownian force is modeled according to the method described in Ref. 17

$$
\mathrm{F}_{\mathrm{B}}=\zeta \sqrt{\frac{12 \pi \mathrm{k}_{\mathrm{B}} \mu \operatorname{Tr}_{\mathrm{p}}}{\Delta \mathrm{t}}} .
$$

Spheres settling on the substrate surface are considered deposited. Figure 2 plots the simulated number of spheres deposited onto the substrate versus settling time for spheres of different sizes.

It is evident that the sedimentation rate is heavily dependent on dimensions. The $990 \mathrm{~nm}$ diameter spheres have the highest initial deposition rates, whereby most of the spheres adhere to the substrate within $10 \mathrm{~min}$, resulting in a sharp decrease of the deposition rate in the subsequent period. On the other hand, the deposition rate for the smaller spheres is slower but remains nearly constant within the simulated time range. Based on the results three factors that affect the sedimentation are identified: the settling time before freezing, as well as the dimensions and concentrations of the spheres.

To verify the predicted deposition behavior based on simulations, coatings using spheres with diameters of 120,490 , and $990 \mathrm{~nm}$ are fabricated via the proposed freeze drying method. The corresponding volume fractions of the spheres are $0.08 \%, 0.14 \%$ and $0.07 \%$, respectively. For each dimension, three settling times of 0,10 , and 30 min have been

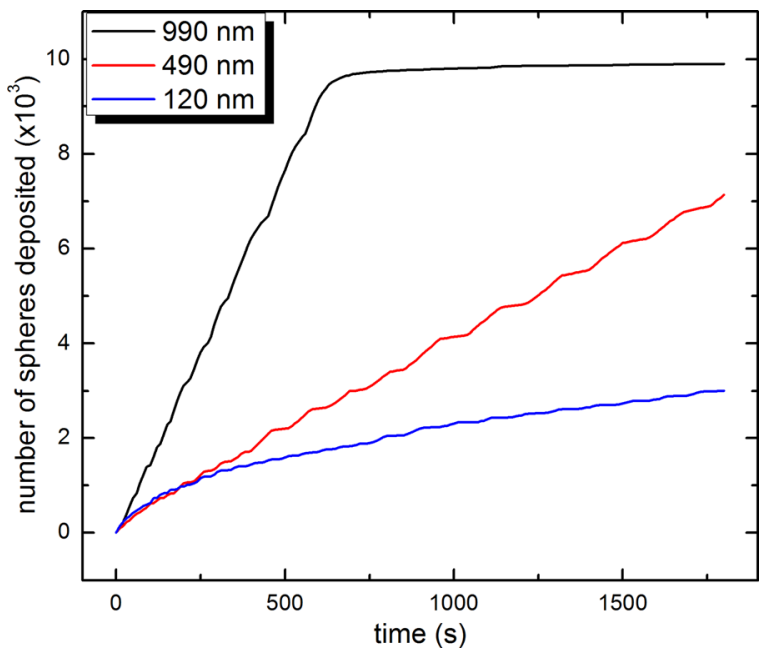

FIG. 2. (Color online) Simulated deposition behavior of spheres of different diameters. 


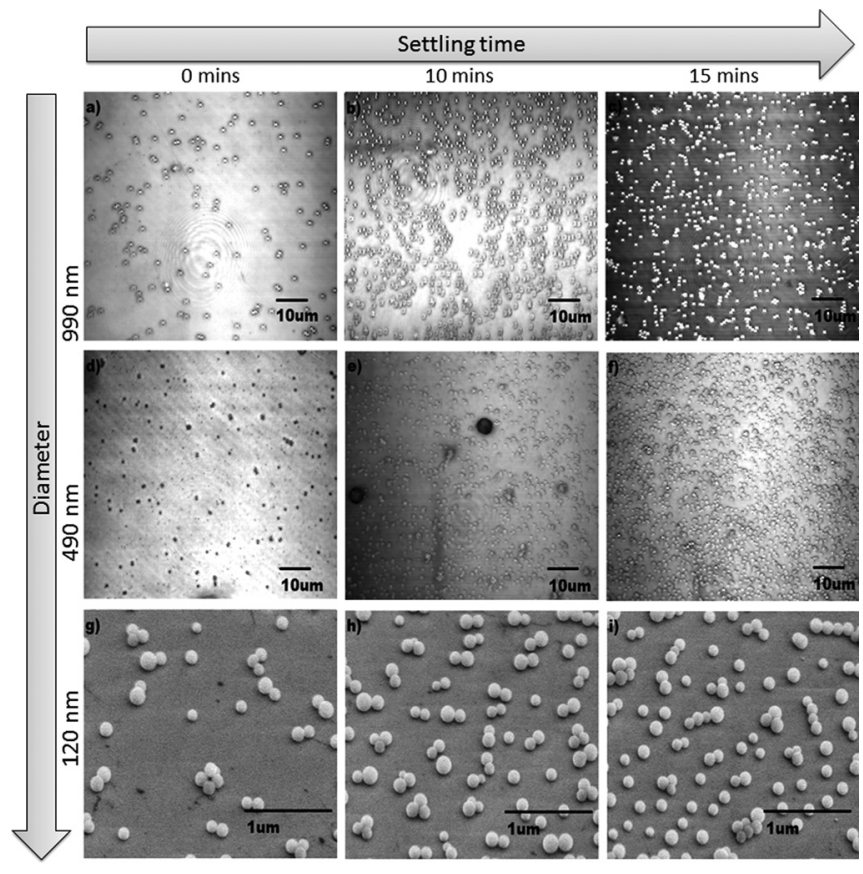

FIG. 3. Sphere coatings prepared by freeze drying of different diameters and settling times.

tested. SEM images of the respective coatings are shown in Fig. 3, while the estimated densities are tabulated in Table I.

Based on the data in Table I, the trends of coated sphere densities as functions of settling time for the 490 and $990 \mathrm{~nm}$ spheres agree well with simulations; the same, however, cannot be said for the $120 \mathrm{~nm}$ spheres. Simulation predicts a continuous increase of density with increasing settling time, while experimental data indicate that the density remains nearly unchanged after $10 \mathrm{~min}$. This is attributed to surface charge effect, which will be further discussed in Sec. III B.

\section{B. Density control}

The factors that control the densities of the coatings are the same factors that affect the overall deposition process. By varying the settling time or the initial concentration (by dilution with DI water) of spheres, monolayers from very low coverage ratio to almost close-packing can be achieved for the 490 and $990 \mathrm{~nm}$ diameter spheres. However, the same cannot be achieved for the $120 \mathrm{~nm}$.

The coating of $120 \mathrm{~nm}$ spheres, as shown in the FE-SEM image of Fig. 4, has a sphere density of $2 \times 10^{9} / \mathrm{cm}^{2}$, corresponding to a coverage ratio of about $25 \%$. Further increase of the settling time or concentration only results more defects being formed, which are mainly agglomerations of spheres as shown in Fig. 4. The remaining spheres are well-

TABLE I. Estimated densities of spheres under different coating conditions.

\begin{tabular}{lccc}
\hline \hline Diameter $(\mathrm{nm})$ & $0 \mathrm{~min}$ & Settling time $10 \mathrm{~min}$ & $30 \mathrm{~min}$ \\
\hline 990 & $2.21 \times 10^{6} / \mathrm{cm}^{2}$ & $1.24 \times 10^{7} / \mathrm{cm}^{2}$ & $1.25 \times 10^{7} / \mathrm{cm}^{2}$ \\
490 & $1.99 \times 10^{6} / \mathrm{cm}^{2}$ & $1.62 \times 10^{7} / \mathrm{cm}^{2}$ & $5.27 \times 10^{7} / \mathrm{cm}^{2}$ \\
120 & $3.25 \times 10^{8} / \mathrm{cm}^{2}$ & $1.60 \times 10^{9} / \mathrm{cm}^{2}$ & $1.70 \times 10^{9} / \mathrm{cm}^{2}$ \\
\hline \hline
\end{tabular}

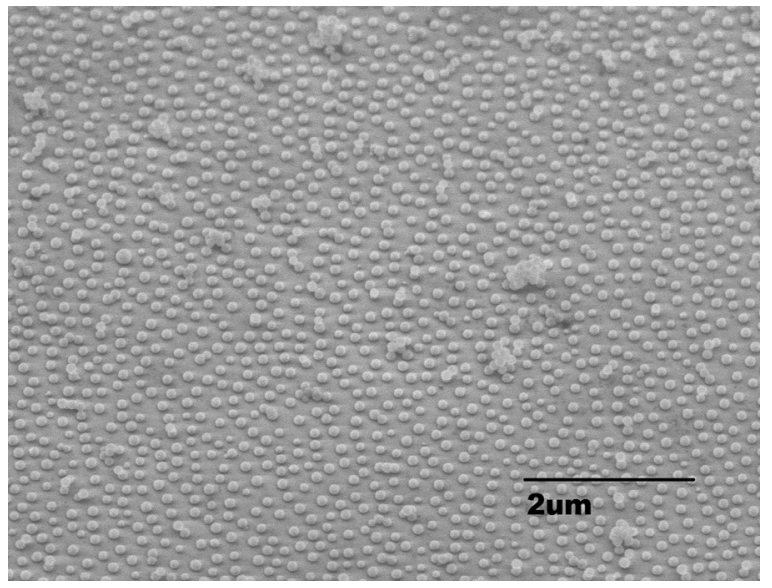

FIG. 4. FE-SEM image showing a $120 \mathrm{~nm}$ sphere coating of the maximum achievable sphere density of $2 \times 10^{9} / \mathrm{cm}^{2}$.

separated, suggesting of the presence of repulsive forces between them. It is reasonable for spheres of such small dimensions to be surface-charged to remain stable in colloidal form, providing repulsive force which also prevents further deposition of spheres. As a result, a coating density limit exists for the $120 \mathrm{~nm}$ spheres, which would also be true for smaller spheres in general.

\section{Defect formation and defect-reducing measures}

Defects refer to agglomerations of spheres in this context. There are three possible origins of defect formation: agglomerations originally existing in the colloid, agglomerations formed during coating and agglomerations formed during storage. The first kind is not within control and thus will not be discussed further.

The FE-SEM image of Fig. 5 shows large agglomerations of $120 \mathrm{~nm}$ spheres over the substrate (sphere volume fraction $0.08 \%$ and settling time $15 \mathrm{~min}$ ). The highly nonuniform distribution of agglomerations suggests that some of the spheres have traveled long distances laterally before gathering into an agglomeration. This is only possible for spheres far away (in the vertical direction) from the substrate, because spheres

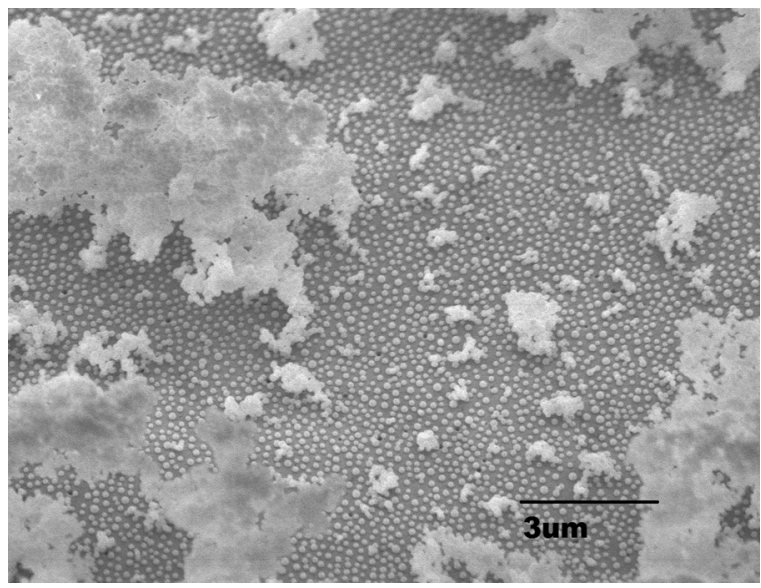

FIG. 5. FE-SEM image showing large agglomerations of $120 \mathrm{~nm}$ diameter spheres. 



FIG. 6. Confocal microscope images showing freeze dry coating of different conditions. (a) Sphere with $990 \mathrm{~nm}$ diameter, $0.07 \%$ volume fraction, 0 min settling time. (b) Sphere with $490 \mathrm{~nm}$ diameter, $0.14 \%$ volume fraction, 0 min settling time. (c) Sphere with $990 \mathrm{~nm}$ diameter, $1 \%$ volume fraction, 0 min settling time. (d) Sphere with $490 \mathrm{~nm}$ diameter, $2 \%$ volume fraction, and 0 min settling time.

near to the surface do not have the same levels of flexibilities or travel range before settling on the substrate. This kind of agglomerations can be effectively removed by three methods.

First, given that the agglomerated spheres originate far away from the substrate surface, agglomeration can be reduced by reducing the number of such spheres. Increasing the settling time is identified as an effective measure, particularly for the 490 and $990 \mathrm{~nm}$ diameter spheres. As explained in Sec. III B, the coating density increases with increasing settling time. Since the total number of spheres is fixed, the amount of suspended spheres will be reduced if more spheres are deposited.

The second method is to reduce the maximum distance between the spheres and the substrate, that is, to thin down the water layer. If all the spheres are close enough to the substrate, instead of traveling a long distance to aggregate, they are more likely to be pulled directly to the substrate during freeze drying. The proposition is verified with the 490 and $990 \mathrm{~nm}$ diameter spheres, with volume fractions of $2 \%$ and $1 \%$, respectively; the settling time is $0 \mathrm{~min}$. The thickness of the water layers is estimated at $\sim 80 \mu \mathrm{m}$, achieved by covering the substrate with a microslide. The results are compared with those coated under regular condition, that is, $\sim 0.4 \mathrm{~mm}$ of water layer, also with 0 min settling time.

As shown in Fig. 6, large-scale agglomerations can be effectively eliminated by thinning down the water layer, offering high-quality coatings with high sphere densities and low defects, even without settling. For $120 \mathrm{~nm}$ diameter spheres, the thinning down of the water layer can avoid large agglomerations, but smaller-scale agglomerations are still present, probably due to the fact that the water layer is still too thick for spheres of such sizes.

Incidentally, it is also found that adhesion between the substrate and the agglomerations is weaker than those between the substrate and the lone spheres. As a result, most of the agglomerations can be removed with a nitrogen gas stream without affecting the coating. In fact, the coating depicted in Fig. 4 represents an example of agglomeration stripping.

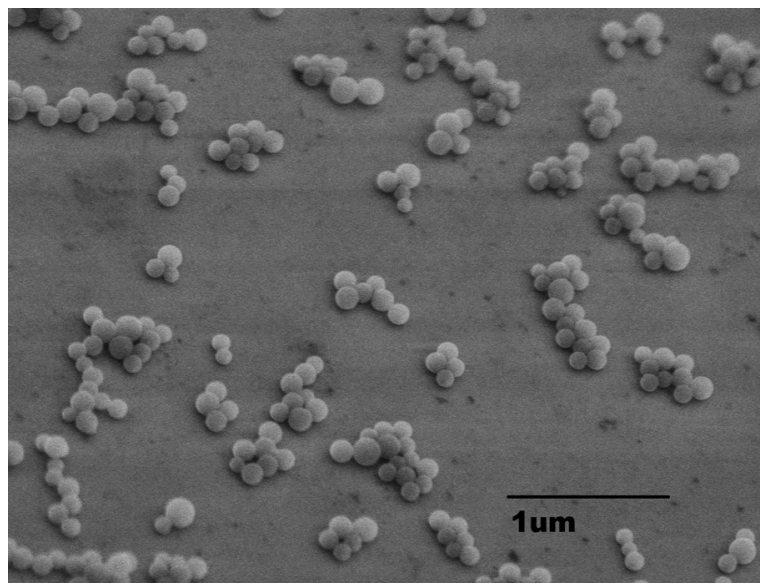

FIG. 7. FE-SEM image showing the aggregation of spheres in a high humidity environment. 

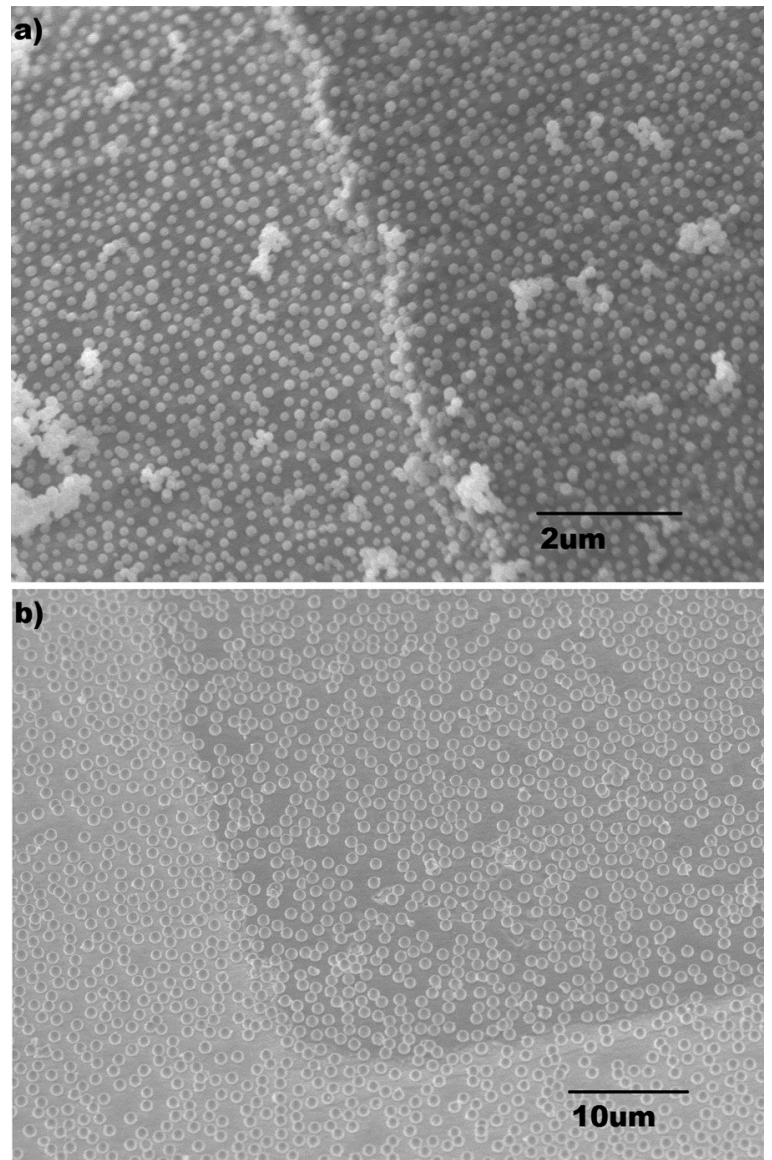

FIG. 8. FE-SEM images showing freeze dry coating on a $500 \mathrm{~nm}$-high mesa structure of (a) $120 \mathrm{~nm}$ and (b) $990 \mathrm{~nm}$ diameter spheres.

Agglomerations can also be formed postcoating. It is observed that the coating is very sensitive to the relative humidity (RH), especially for the $120 \mathrm{~nm}$ diameter coating. Exposure to $\mathrm{RH}>40 \%$ for minutes will invariably lead to the gathering of spheres, as shown in Fig. 7. Therefore, the coatings must be stored in a dry environment before the next process step.

Finally, the robustness of this freeze drying coating method is highlighted. From the FE-SEM image of Fig. 7, the poor uniformity of the $120 \mathrm{~nm}$ diameter spheres is evident $( \pm 40 \mathrm{~nm})$. Figures 8(a) and 8(b) show coatings of 120 and $990 \mathrm{~nm}$ diameter spheres across a mesa structure with a step-height of $500 \mathrm{~nm}$. It is observed that the coating quality is affected neither by the step nor the dimensional nonuniformities of the spheres. Note that these two factors are usually causes of multilayer defects in conventional coating methods, especially spin-coating.

\section{CONCLUSION}

A simple, efficient, and robust way for ncp monolayer coating of nanospheres based on freeze drying has been demonstrated. The coating mechanism, density control, and defect control have discussed. Ncp monolayers of spheres with diameters ranging from 120 to $990 \mathrm{~nm}$ have been achieved.

\section{ACKNOWLEDGMENT}

This work was supported by a General Research Fund (HKU711212E) of the Research Grant Council of Hong Kong.

${ }^{1}$ C. L. Haynes and R. P. Van Duyne, J. Phys. Chem. B 105, 5599 (2001).

${ }^{2}$ S. M. Yang, S. G. Jang, D. G. Choi, S. Kim, and H. K. Yu, Small 2, 458 (2006).

${ }^{3}$ X. Ye and L. Qi, Nano Today 6, 608 (2011).

${ }^{4}$ A. P. Sommer, M. Ben-Moshe, and S. Magdassi, J. Phys. Chem. B 108, 8 (2004).

${ }^{5}$ J. Perelaer, P. J. Smith, C. E. Hendriks, A. M. J. van den Berg, and U. S. Schubert, Soft Matter 4, 1072 (2008).

${ }^{6}$ R. G. Shimmin, A. J. DiMauro, and P. V. Braun, Langmuir 22, 6507 (2006).

${ }^{7}$ D. Xia, A. Biswas, D. Li, and S. R. J. Brueck, Adv. Mater. 16, 1427 (2004).

${ }^{8}$ M. Szekeres, O. Kamalin, R. A. Schoonheydt, K. Wostyn, K. Clays, A. Persoons, and I. Dékány, J. Mater. Chem. 12, 3268 (2002).

${ }^{9}$ M. Bandilla, A. Zimdars, S. Neugebauer, M. Motz, W. Schuhmann, and G. Hartwich, Anal. Bioanal. Chem. 398, 2617 (2010).

${ }^{10}$ J. A. Scholl, L. K. Ai, and J. A. Dionne, Nature 483, 421 (2012).

${ }^{11}$ H. J. Fan, B. Fuhrmann, R. Scholz, F. Syrowatka, A. Dadgar, A. Krost, and M. Zacharias, J. Cryst. Growth 287, 34 (2006).

${ }^{12}$ K. Peng, M. Zhang, A. Lu, N. B. Wong, R. Zhang, and S. T. Lee, Appl. Phys. Lett. 90, 163123 (2007).

${ }^{13}$ K. H. Li and H. W. Choi, J. Appl. Phys. 109, 023107 (2011).

${ }^{14}$ P. Jiang and M. J. McFarland, J. Am. Chem. Soc. 126, 13778 (2004).

${ }^{15}$ P. Jiang, T. Prasad, M. J. McFarland, and V. L. Colvin, Appl. Phys. Lett. 89, 011908 (2006).

${ }^{16}$ T. Lohmüller et al., J. Micromech. Microeng. 18, 115011 (2008).

${ }^{17}$ M. M. Kim and A. L. Zydney, J. Colloid Interface Sci. 269, 425 (2004). 